\title{
Elementos gráficos y construcción de objetos virtuales en ambientes informáticos: Reflexiones en torno a la educación
}

\author{
Edward Carvajal Arciniegas ${ }^{1}$ \\ Universidad de San Buenaventura (Colombia)
}

Recibido: septiembre 9 de 2015. Revisado: noviembre 27 de 2015. Aceptado: julio 12 de 2016

Referencia formato APA: Carvajal, E. (2016). Elementos gráficos y construcción de objetos virtuales en ambientes informáticos: Reflexiones en torno a la educación. Rev. Guillermo de Ockham, 14(2), 131-141. doi: http://dx.doi.org/10.21500/22563202.2563

\section{Resumen}

Este artículo hace una exploración a la forma como se seleccionan algunos elementos gráficos para su posterior incorporación en la construcción de objetos virtuales (app), con base en la interacción de sujetos en la virtualidad. Esta indagación hizo uso de la observación no participante y de entrevistas estructuradas con guía, a un grupo de estudiantes de ingeniería multimedia integrantes de un semillero en tecnología web. A partir de este estudio se reconoció un proceso de mediación en la creación de objetos virtuales y se evidenciaron otras formas de acceso a la información mediante comunidades de desarrollo con la ayuda de dispositivos móviles, lo cual generó reflexiones acerca de la tensión entre los procesos educativos y la virtualidad en internet causada por la libertad con que la información viaja por la red.

Palabras clave: Virtualización, mediación, objetos virtuales, educación

\section{Graphic elements and construction of virtual objects in computer-based fields: Reflections regarding education Abstract}

This paper realizes an exploration to the form of how some graphical elements are chosen for their incorporation in the construction of virtual objects (app) bearing the interaction of subjects in the virtuality. This research was done, using the nonparticipant observation and interviews structured with a guide, to a group of students of multimedia engineering, members of a seedbed in web technology. From this study, we recognized a process of mediation in the creation of virtual objects and to demonstrate other forms of access to the information through communities of development with mobile devices; which generated reflections about the existing tension between the educational processes and the virtuality in Internet, caused by the way information is freely shared online.

Keywords: Virtualization, mediation, virtual objects, education

* El presente artículo incorpora los resultados de la investigación Virtualizacción: la interacción de los sujetos en la construcción de objetos virtuales. Universidad de San Buenaventura Cali. Fecha de inicio: marzo, 2014. Fecha final: mayo, 2015.

1. Magíster en Educación: Desarrollo Humano de la Universidad de San Buenaventura Cali. E-mail: ecarvajal@usbcali.edu.co 


\section{Elementos gráficos e construção de objetos virtuais em ambientes informáticos: Reflexões sobre á educação}

\section{Resumo}

Este artigo faz uma exploração á forma como são seleccionados algums elementos gráficos para sua posterior incorporaçáo na construçáo de objetos virtuais (app), com base na interaçáo de sujetos em virtualidade. Esta pesquisa fez uso da observação não-participante e de entrevistas estruturadas com um guía, a um grupo de estudantes de engenharia ultimedia membros de um semillero em tecnología web. A partir deste estudo foi reconhecido um processo de mediação na na criação de objetos virtuais e outras formas de acesso à informação através de comunidades de desenvolvimento com a ajuda de dispositivos móveis, gerando reflexóes sobre a tensão entre processos educativos e a virtualidade causada pela liberdade com que a informação viaja através da rede.

Palavras-chave: Virtualização, mediação, objetos virtuais, educação

\section{Introducción}

La tecnología web ejerce notable influencia sobre las personas, quienes intercambian información y generan relaciones intersubjetivas a partir de los objetos virtualizados que se encuentran en los ambientes informáticos generados en internet. Esto evidencia su carácter de construcción social y resalta la importancia de la iconografía en estas plataformas y la necesidad de encontrar respuestas a los retos que la virtualidad genera con respecto a la comunicación y a la educación en estos medios digitales.

Estudios previos han destacado la importancia de las aplicaciones educativas que se utilizan a través de dispositivos electrónicos (mobile learning), por ser herramientas importantes para apoyar la educación a distancia gracias a que la conectividad por internet le permite un rápido acceso de la información. Esto brinda mayores beneficios en cuanto a movilidad, acceso y disponibilidad de los contenidos educativos (De la peña \& Burgos, 2015). Así mismo, Bautista y Méndez (2015) hacen énfasis en que la incorporación de las TIC en el aula va más allá del uso de artefactos novedosos y afirman que la importancia de estos elementos radica en la creación de otras formas de comunicación y circulación de la información; lo que genera espacios colaborativos que ayudan a la comprensión y producción de textos a partir de la hipertextualidad y la interactividad.

También se afirma que la imagen digital debe ser tenida en cuenta como elemento importante en la comunicación, dada la relación que se establece entre el usuario y el ordenador a partir de las interfaces gráficas de usuario ${ }^{2}$ (Alberdi, 2014). De la misma manera, Buján (2010) ha hecho énfasis en este mismo elemento (interfaz gráfica), pero en alusión a la importancia de los nexos intersubjetivos que se establecen en los entornos virtuales de aprendizaje. El término "intersubjetivo" ha sido puesto en relevancia por González y Hernández (2008) cuando afirman que las interfaces de usuario de las aplicaciones tecnológicas, además de ser herramientas para el aprendizaje, también son elementos importantes para la comunicación en los procesos educativos y en la interacción.

Específicamente, Roos y Gao (2015), llevan a cabo un investigación en el sector salud en la cual destacan la interfaz gráfica de usuario como uno de los elementos más importantes de las aplicaciones móviles gracias a que rompen las barreras del lenguaje y en las cuales factores como los usuarios, la simplicidad, la disposición de los elementos, los colores el texto y la accesibilidad, son importantes para la creación de estas aplicaciones. Lo que concuerda con Chun-Heng y Hou (2015), quienes destacan el aumento de los teléfonos inteligentes y la necesidad de crear íconos que representen las aplicaciones de una manera atractiva en las tiendas de aplicaciones. El estudio también concluye que es fundamental pensar en el usuario final a la hora de elaborar la interfaz gráfica, no solo en el aspecto funcional básico, sino también en cuanto a la necesidad de tener en cuenta el sentir o el gusto estético de los usuarios.

A partir de estas perspectivas, la investigación que se presenta a continuación retoma algunos de los elementos anteriores para enfocarse en un proceso del cual no se tiene referencia y apunta a evidenciar los elementos gráficos utilizados en la construcción de un objeto virtual; es decir, le provee un enfoque distinto al no analizar el funcionamiento o las relaciones que se generan, sino enfatizar en

2. La interfaz gráfica de usuario es un programa o entorno que gestiona la interacción con el usuario basándose en relaciones visuales como iconos, menús o un puntero. 
su construcción, debido a que estos elementos simbólicos permiten la interacción y el uso de los objetos en la red. De esta manera, la investigación describe algunos elementos gráficos que se privilegian en la construcción de un objeto virtual elaborado para ambientes informáticos (app) y reconoce la forma de interacción de los sujetos en la red para efectuar la búsqueda de dichos elementos.

En esta investigación se hace un análisis inductivo mediante la relación directa con los sujetos que elaboran interfaces gráficas para objetos virtuales, basado en la premisa de la metodología del interaccionismo simbólico (Blumer, 1982) quien afirma que en términos metodológicos no es necesario formular hipótesis para las investigaciones. Sin embargo, parte de la idea de que los seres humanos no se limitan solo a reaccionar ante estímulos, sino que también interpretan y definen -o redefinen-acciones de otros, dando así respuesta a estos de acuerdo con lo que signifiquen estas acciones para ellos. Por eso, la interacción humana se convierte en un medio para la comunicación a través de símbolos y su significado es el producto del proceso entre los individuos, el cual se "manipula" y modifica de acuerdo al proceso de interpretación que realizan los sujetos cuando se enfrenta a las cosas (PUJ, s.f.).

Así, se considera pertinente explorar la manera como las relaciones intersubjetivas permiten crear signos para la comunicación entre sujetos en los ambientes informáticos y establecer nexos entre los elementos gráficos usados en la construcción de objetos virtuales. Estos elementos resaltan la importancia de investigar en tecnologías web, las cuales incorporan una multiplicidad de fenómenos que ayudan a que internet se convierta en una herramienta importante para explorar el mundo. Esta tecnología provee un medio de diseminación de información que requiere la interacción entre sujetos -o entre sujetos y objetos- para acceder a los datos que circulan en la red.

Estos datos -que no están centralizados- suministran a los sujetos herramientas para la construcción de conocimiento y les exigen destrezas para el acceso a la información. Incluso, se hace necesario brindar importancia a la función crítica del sujeto para filtrarla cuando se utilizan estos espacios con fines educativos, ya que es posible estar desinformado pese a la gran cantidad de documentos que circulan en internet (Tedesco \& García, 2007); o como lo afirma Baudrillard (1984), se generan dudas con respecto a los datos presentados por los medios de comunicación, no porque se carezca de información, sino por su exceso.

Este tiempo de lo virtual (Virilio, 1999), permite la circulación de gran cantidad de información que se genera gracias a que los navegantes de internet son potenciales "alimentadores" de esta red de datos cuando ingresan imágenes y videos o cuando hacen comentarios en un sitio web, ya que este lugar se nutre de las relaciones entre los sujetos, lo que permite la comunicación a través de dispositivos móviles o aparatos electrónicos. De acuerdo con Martín-Barbero (1987), se trata de procesos de mediación, porque es a partir de la participación humana que se modifican los mensajes; esto en referencia a que los dispositivos son medios o agentes en estos procesos y su función es posibilitar el encuentro o el intercambio de información; sin embargo, los sujetos son mediadores dado que a partir de su interacción producen esas relaciones y hacen posible que la información se visibilice en las redes digitales.

Una opción para abordar la mediación en la tecnología web apunta al uso que se les brinda a estos espacios y a las distintas formas de interacción a partir de los objetos elaborados en la virtualidad, haciendo énfasis en las ventajas o los desafíos que se presentan. Sin embargo, otra forma de aproximarse-lo cual es objeto de este estudio-es ampliar la mirada hacia la construcción de estos objetos virtuales de manera que no se analicen según su uso o aprovechamiento, sino a partir de los elementos utilizados en su elaboración. Para esto, se deben tener en cuenta los sujetos que los construyen y profundizar en la forma como estas tecnologías permiten exteriorizar u objetivar procesos sociales o comunicativos y la manera como estas construcciones de objetos virtuales deben estar en armonía con los lenguajes, las formas y los discursos que circulan en la red, mediante una aproximación a los símbolos que se incluyen en los objetos para facultar la comunicación entre sujetos.

Se hace énfasis en los objetos toda vez que son producto de la creación humana y su función o uso puede transformarse o desecharse en la interacción y en la experiencia con los sujetos en la sociedad al establecer otras formas de relación entre los individuos (Giraldo, 2009). Incluso, las nuevas relaciones pueden propiciar la creación de nuevos objetos y es el objeto el elemento que sustenta lo virtual porque no se desgasta con el uso, sino que, por el contrario, equilibra el cúmulo de fuerzas o las tensiones que se generan en el grupo y permite el paso bilateral entre lo público y lo privado (Lévy, 1998).

\section{Virtualización}

En una primera aproximación, Lévy (1998) indica que lo virtual no es semejante a lo falso, a lo ilusorio o a lo imaginario, ni está opuesto a lo real, sino que es un 
elemento dinámico que fomenta los procesos creativos. También nos recuerda que la palabra virtual procede del latín virtualis, la cual a su vez, es un derivado de virtus, que significa fuerza o potencia, $y$ resalta que lo virtual existe de manera latente, pero no ha sido actualizado.

A partir de esta teoría, se define de manera general la virtualización como un proceso que posibilita el paso de lo real a lo actual o a lo virtual. Por lo tanto, es un proceso que no consiste en convertir la realidad en un cúmulo de probabilidades, sino que es necesario mirar de manera distinta el objeto o elemento virtualizado, para que en lugar de encontrar soluciones predefinidas se convierta en un elemento complejo, algo que no está definido en parte alguna, una problemática general desterritorializada y colectivizada (Lévy, 1998).

Siendo así, podemos establecer diferencias entre la virtualidad como potencia del sujeto y los procesos de virtualización. Esta virtualización permite determinar una nueva espacio-temporalidad de un proceso o de un objeto real con base en dos características principales: la desterritorialización y el efecto Moebius. La primera se evidencia como una separación entre el espacio físico y la temporalidad; por tal razón, cuando los sujetos, las actividades o algún elemento se virtualizan, se genera un proceso de desprendimiento entre el lugar físico donde estos confluyen y el tiempo en que se puede efectuar el encuentro. La segunda característica -efecto Moebiusrepresenta el paso persistente del interior al exterior y viceversa, y hace referencia al tránsito constante entre lo público y lo privado en el que se resalta la importancia del sujeto en estas relaciones que forman parte de los procesos de virtualización y la forma como estos nexos intersubjetivos, -simbolizados en el Efecto Moebius-dotan de sentido las conexiones entre los sujetos.

La analogía entre el efecto Moebius y los procesos intersubjetivos, permite enfatizar que la sociedad construye la realidad a través de las prácticas sociales en los diversos entornos (Berger \& Luckmann, 2001), lo que preserva el significado de las objetivaciones humanas dentro de la experiencia compartida en la circulación de los discursos y da cuenta de que la virtualidad no es un fenómeno exclusivo del manejo de las relaciones a través de la conexión por medio de dispositivos electrónicos o de la incursión de internet, sino que estas acciones se generan en la vida cotidiana. Por ello, para esta indagación es necesario darles importancia a los elementos tecnológicos que influencian a los sujetos en los diversos sitios web. Si bien, para Schutz (Belvedere, 2013), la intersubjetividad se genera en el contacto "cara a cara", es importante establecer qué percepción resulta de las formas de comunicación efectuada a través de dispositivos móviles con ayuda de la conectividad y la interacción en "tiempo real", ello sobre la base de que este tipo de interacción se hace a través de objetos que han sido virtualizados.

Esta investigación se enfoca, entonces, en los sujetos para explorar lo que sucede cuando un grupo de personas requiere seleccionar mediante la interacción en la virtualidad, elementos gráficos para la construcción de un objeto virtual que será utilizado en un ambiente informático.

\section{Método}

\section{Diseño}

Se trata de una investigación cualitativa con un alcance exploratorio (Hernández, Fernández \& Baptista, 2010), en la cual se llevó a cabo una indagación sobre la forma como los sujetos integrantes de un semillero de investigación en tecnología web hacían la elección de elementos gráficos para ser utilizados en la construcción una aplicación informática (app), con base en su interacción en ambientes informáticos.

Se efectuó una observación no participante en las reuniones grupales del semillero y una entrevista estructurada con guía (Bonilla-Castro \& Rodríguez, 1997) a cada integrante del semillero. Esta observación se hizo de acuerdo con dos elementos primordiales del interaccionismo simbólico: el objeto y la interacción social (Blumer, 1982), el cual postula que el ser humano define sus actos hacia las cosas (objetos) en función de lo que estas signifiquen para él, lo que se produce como consecuencia de la interacción social. Esto atribuye gran importancia al significado de los objetos y a la interacción como procesos humanos primordiales toda vez que se crean de manera colectiva.

Para este análisis se tuvo en cuenta que el interés del interaccionismo simbólico se centra en la idea de que la influencia del significado de los símbolos surge no solo de los procesos mentales, sino también de la interacción social, por cual el significado no es un elemento subjetivo sino una reacción objetiva que se aprende para luego hacerse general (Carabaña \& Lamo de Espinoza, 1978). Sobre la base de estos aspectos, se eligieron dos categorías de análisis: 1. elementos gráficos para la construcción de objetos virtuales en ambientes informáticos, y 2 . interacción de los sujetos en ambientes informáticos.

\section{Participantes}

La investigación tuvo como grupo de observación a cuatro personas -tres estudiantes y un docente líder- per- 
tenecientes a un semillero de investigación en tecnología web, adscrito al programa de Ingeniería Multimedia de una universidad privada de la ciudad Cali, quienes hacen parte de un proceso de creación de una aplicación informática (app) que servirá como canal de comunicación e información en su comunidad educativa.

Se eligió este grupo de personas para lograr describir el espacio de los sujetos que están realizando el proceso de elección de los elementos gráficos para la creación de un objeto virtual (app) en el lugar donde se elabora esta apropiación; donde, a través de la interacción por medio de ambientes informáticos, se analizan símbolos e íconos para crear unos nuevos que puedan ser entendidos por los demás usuarios de la aplicación.

\section{Instrumentos}

Se hizo uso de la observación no participante (BonillaCastro y Rodríguez, 1997) a los integrantes del semillero de investigación, quienes hacían entregas parciales de sus indagaciones sobre los elementos gráficos necesarios en la construcción de objetos virtuales y los diversos elementos técnicos tenidos en cuenta para la construcción de la aplicación (app), a través de la metodología Scrum. ${ }^{3} \mathrm{La}$ información de sus entregas parciales fueron compiladas en el documento Pocket College, ${ }^{4}$ utilizado como parte de esta investigación. De esta manera se llevó a cabo la exploración de algunos elementos importantes y se focalizó una entrevista personal a los tres estudiantes ${ }^{5}$ miembros del semillero de investigación, con el fin de obtener información acerca de sus conocimientos e indagar sobre la manera como organizan y orientan su comportamiento en el proceso de construcción de objetos virtuales.

\section{Procedimiento de aplicación}

Durante diez sesiones de una hora semanal desarrolladas entre agosto y octubre de 2014, se observaron las reuniones del semillero de investigación y en ellas se evidenciaban las implicaciones de desarrollar aplicativos para los sistemas operativos iOS y Android, además de rastrearse las aplicaciones más populares para conocer sus usos frecuentes, su interacción y los inconvenientes reportados por los usuarios. Se hizo también referencia a los dispositivos más utilizados, su sistema operativo, el tipo de iconografía estandarizada, la tipografía y los colores que mejor se adaptaban a los tamaños y a la resolución de pantalla de estos dispositivos.

Adicionalmente, en el mes de diciembre de 2014 se hizo una entrevista estructurada con una guía (BonillaCastro \& Rodríguez, 1997) de cuarenta y tres preguntas a cada uno de los tres estudiantes que integraban el semillero. Las primeras dieciocho preguntas se enfocaron en reconocer datos específicos de interés para la universidad y el semillero al que pertenecen, con el fin de fortalecer temas institucionales. Las siguientes diez buscaban referenciar los fundamentos teóricos que utilizaban para abordar el problema de la virtualidad (estas preguntas iniciales tienen como intención una segunda fase de este estudio). Las siete preguntas siguientes hacen referencia a determinados elementos visuales requeridos para el proceso de construcción de interfaces gráficas, con base en la importancia de los dispositivos y la conectividad. Las últimas ocho preguntas se enfocaron en percibir su experiencia para obtener dicha información mediante la interacción en sitios web o en medios digitales.

\section{Procedimiento de medición}

Durante la observación no participante se describieron los elementos gráficos que se privilegiaban en dicha construcción de la aplicación informática (app) y se reconoció la forma en que los integrantes del semillero obtenían dicha información. A partir de este ejercicio, se efectuó una categorización inductiva (Bonilla-Castro \& Rodríguez, 1997), en la que se establecieron dos categorías para el análisis: 1. elementos gráficos para la construcción de objetos virtuales en ambientes informáticos, que enfatiza los elementos gráficos como la tipografía, la paleta de colores, la iconografía, la navegación, la usabilidad y la estructura, que se tienen en cuenta en la construcción de un objeto virtual ( $a p p)$; y 2. la interacción de sujetos en ambientes informáticos, en la que se entiende interacción como la acción ejercida recíprocamente entre dos o más sujetos. Es decir, observar la manera como se orienta la conducta hacia otros a partir de la relación surgida en los ambientes informáticos, de manera que puedan hacer la elección de algunos elementos gráficos en la construcción de un objeto virtual $(a p p)$.

3. Consiste en un proceso basado en prácticas y funciones apoyadas en el trabajo colaborativo, en el que se ejecutan de manera regular una serie de reuniones para trabajar en equipo, con el objetivo de lograr el mejor resultado posible de un proyecto, de acuerdo con metas específicas.

4. El Pocket College es un documento creado por el semillero de investigación como hoja de ruta acerca de la forma como se construye una app. Este documento incluye algunos elementos tenidos en cuenta en la creación de la interfaz gráfica de usuario, su terminología y algunos ejemplos que hacen referencia a la creación de app en otras universidades.

5. No se incluyó en esta entrevista al docente líder del grupo dado que sus funciones en el semillero eran distintas a las de los estudiantes de ingeniería multimedia. 
Una vez formuladas estas categorías, se efectuaron y grabaron en medio digital las entrevistas a cada miembro del semillero y se establece un orden de preguntas referentes al conocimiento teórico de los asuntos tratados y su forma de acceder a la información, de manera que se determinó la forma como se seleccionaban y priorizaban algunos elementos gráficos utilizados en la construcción de objetos virtuales.

\section{Procedimiento de análisis}

Se hizo un análisis descriptivo (Hernández, Fernández \& Baptista, 2010) de las notas tomadas durante la observación no participante y de la información contenida en el Pocket College. Posteriormente, se seleccionaron los elementos gráficos más relevantes en la construcción del objeto virtual y se hizo una bitácora con esta descripción para conocer la importancia de estos elementos en el diseño de la aplicación. Los datos se ubicaron en cada una de las categorías.

Adicionalmente, se transcribieron las entrevistas y se hicieron listas de conteo agrupando las respuestas por el tipo de repeticiones que estaban referidas a la forma como obtenían la información por medio de la interacción y se establecieron cadenas lógicas de evidencias (Bonilla-Castro \& Rodríguez, 1997). Se presentaron las dinámicas de acceso a dicha información y se establecieron los elementos y sus relaciones.

Finalmente, se estableció una matriz descriptiva (Bonilla-Castro \& Rodríguez, 1997) (Tabla 1) en la cual se incluyeron los comentarios similares que hacían referencia a cada categoría y se generaron los datos según sus coincidencias.

\section{Resultados}

El proceso de construcción de aplicaciones (app) puede dividirse en dos equipos de trabajo: BackEnd, a cargo de los procesos de ingeniería de software, y FrontEnd, relativo al componente de diseño. Ambos grupos analizan los diversos sistemas operativos que dan soporte a los dispositivos móviles y revisan los elementos que permiten la creación de la aplicación, incluidas su estructura técnica y la usabilidad. ${ }^{6}$

Al comenzar el proceso de recolección de información no se tienen en cuenta los aspectos gráficos de la aplicación sino los funcionales y técnicos, de manera que se puedan evidenciar inconvenientes y definir soluciones antes del desarrollo. Para esto, los desarrolladores estructuran un documento que incorpora las funcionalidades de la aplicación, sus detalles técnicos, el cliente objetivo y los avances tecnológicos con los que se llevará a cabo el proyecto.

Se destaca la importancia de los dispositivos móviles (celulares y tabletas) para el desarrollo de aplicaciones web, dada la necesidad de analizar las probabilidades en cuanto a los sistemas operativos y sus características particulares, así como las diversas marcas. Este último aspecto dificulta o hace más riguroso el análisis por parte de los integrantes del semillero.

Se evidencia que este tipo de desarrollos son factibles de modificarse no solo en cuanto a los avances tecnológicos, sino también de acuerdo con los que los usuarios proponen. De esta manera, una aplicación puede volverse obsoleta por su funcionalidad, pero también por la aceptación de los usuarios.

\section{Elementos gráficos para la construcción de objetos virtuales en ambientes informáticos}

La paleta de colores, la tipografía y la iconografía son algunos de los elementos gráficos tenidos en cuenta para la construcción de la aplicación informática. Estos elementos deben tener criterios de unidad y se hace énfasis en su ubicación en la pantalla del ordenador y la relación entre ellos. Para lograrlo, deben ajustarse a los estándares publicados en los sitios web oficiales de iOS y Android.

Su usabilidad es el aspecto más importante, toda vez que pone en evidencia las jerarquías que se incorporarán en la aplicación y facilita al máximo la experiencia del usuario al permitirle comprender las acciones que debe efectuar con los botones.

Para este tipo de aplicaciones web se muestra una tendencia a utilizar el patrón de diseño Flat design que forma parte de la preferencia gráfica sugerida para la interfaz de usuario de Windows 8 y se utilizan plantillas (themes) que se pueden descargar de los sitios web de los proveedores de sistemas operativos, con lo que aseguran que la aplicación se ajuste a requerimientos técnicos en su desarrollo.

\section{Interacción de sujetos en ambientes informáticos}

Para resolver las dudas referidas a los aspectos técnicos de la aplicación, los integrantes del semillero recurren a comunidades de desarrollo apoyadas en foros, blogs y

6. La usabilidad es la medida de la calidad de la experiencia que tiene un usuario cuando interactúa con un producto o sistema. 
Tabla 1

Matriz descriptiva*E1, E2 y E3 corresponden, respectivamente, a entrevistado 1, entrevistado 2 y entrevistado 3. El contenido de estas entrevistas se transcribió literalmente.

\begin{tabular}{|c|c|c|c|}
\hline Categorías & Aspectos & $\begin{array}{c}\text { Observación no } \\
\text { participante }\end{array}$ & Entrevista estructurada con guía* \\
\hline \multirow{6}{*}{$\begin{array}{l}\text { Elementos gráficos } \\
\text { para la construcción } \\
\text { de objetos virtuales en } \\
\text { ambientes informáticos. }\end{array}$} & $\begin{array}{l}\text { Identificación de } \\
\text { elementos gráficos } \\
\text { de la app. }\end{array}$ & $\begin{array}{l}\text { Paleta de colores, tipografía, } \\
\text { iconografía, navegación, } \\
\text { estructuración (interfaz gráfica } \\
\text { de usuario, usabilidad, look } \\
\text { and fell). }\end{array}$ & $\begin{array}{l}\text { Hay dos formas de dividir el equipo de trabajo: FrontEnd y BackEnd. La primera se } \\
\text { encarga del componente gráfico y la segunda de la programación. } \\
\text { E2: "Entonces hay que tratar de dividir el equipo en dos partes: unos, los que se encargan } \\
\text { del FrontEnd y otros del BackEnd. Una vez que esto está definido, lo que se puede conti- } \\
\text { nuar es el desarrollo y siempre hacer algunas pruebas de usuario". }\end{array}$ \\
\hline & $\begin{array}{l}\text { Criterios de } \\
\text { elección. }\end{array}$ & $\begin{array}{l}\text { Se revisan las tendencias y los } \\
\text { lineamientos de los sitios web } \\
\text { autorizados de las empresas } \\
\text { constructoras del software iOS } \\
\text { o Android. }\end{array}$ & $\begin{array}{l}\text { Es necesario hacer una documentación del proceso para saber cuáles elementos se privile- } \\
\text { gian y de qué manera se resolverían los aspectos técnicos. } \\
\text { "E2: "El proceso nos tomó unos tres o cuatro meses, en el que estuvimos trabajando en } \\
\text { la documentación y cómo iba a estar el proyecto. Lo más importante es definir una idea y } \\
\text { mirar qué se pretende solucionar con esa idea, cuál es la problemática". }\end{array}$ \\
\hline & $\begin{array}{l}\text { Sistemas opera- } \\
\text { tivos. }\end{array}$ & $\begin{array}{l}\text { El análisis de hizo basado en } \\
\text { los sistemas operativos iOS o } \\
\text { Android. }\end{array}$ & \\
\hline & $\begin{array}{l}\text { Dispositivos } \\
\text { móviles. }\end{array}$ & $\begin{array}{l}\text { Es difícil hacer énfasis en algún } \\
\text { dispositivo, porque hay gran } \\
\text { variedad. Se hizo un análisis } \\
\text { genérico de sus elementos para } \\
\text { abarcar la mayor cantidad } \\
\text { de posibilidades en cuanto a } \\
\text { tamańo y marcas. }\end{array}$ & $\begin{array}{l}\text { Se debe tener muy clara la diferencia entre una aplicación para computadores y una para } \\
\text { dispositivos móviles (tabletas, celulares). } \\
\text { E1: "En un celular sabés que tenés un tamaño mínimo por la cuestión de poder tocarlos. } \\
\text { Entonces, según la plataforma o el dispositivo para el que se vaya a hacer, cambia todo". } \\
\text { E3: "Tenés que ver todas las posibilidades. Todas las hipótesis que pensés las debés tener } \\
\text { en cuenta, porque es algo para muchas personas y para diferentes dispositivos". }\end{array}$ \\
\hline & Tendencia gráfica. & $\begin{array}{l}\text { Los usuarios se adecuan a la } \\
\text { tendencia FlatDesign que pro- } \\
\text { viene del diseño de la interfaz } \\
\text { gráfica de Windows } 8 \text { (diseńo } \\
\text { plano, sin volumen) }\end{array}$ & $\begin{array}{l}\text { Los usuarios utilizan como modelo gráfico el FlatDesign y lo ajustan a los requerimientos } \\
\text { de la aplicación, según el cliente. } \\
\text { E2: "Una persona que va a trabajar FrontEnd, debe entender las tendencias de diseño } \\
\text { que se están viendo hoy en día y a partir de eso generar algo creativo, pero dentro de esas } \\
\text { tendencias que se están manejando". }\end{array}$ \\
\hline & $\begin{array}{l}\text { Elementos que se } \\
\text { privilegian. }\end{array}$ & $\begin{array}{l}\text { La usabilidad y el look and fell. } \\
\text { No se revisa un elemento en } \\
\text { particular. Primero se ajusta el } \\
\text { conjunto de elementos. } \\
\text { Privilegian el hecho de tener } \\
\text { en cuenta el usuario final para } \\
\text { que pueda comprender la } \\
\text { aplicación. }\end{array}$ & $\begin{array}{l}\text { Más que un elemento en particular, se privilegia el conjunto (usabilidad); que el usuario } \\
\text { pueda comprender el desarrollo. } \\
\text { Importancia de pensar en los demás para que comprendan los aspectos técnicos de la } \\
\text { aplicación. } \\
\text { E1: "Primero, debemos saber si lo que nosotros estamos planteando es realmente útil o si } \\
\text { lo van a usar; si la gente lo considera necesario. } \\
\text { E2: "Lo que yo espero es que el usuario pueda, de la manera más intuitiva y de la forma } \\
\text { más fácil posible, acceder a la información que él quiere". } \\
\text { "Lo más complicado de crear estos objetos virtuales es salirse de pensar como uno y poner- } \\
\text { se a pensar como podría ser todo el mundo". } \\
\text { E2: "Porque cuando uno está diseńando algo, uno piensa: 'bueno, a mí me gusta mucho } \\
\text { esto'. Pero tiene que tener en cuenta lo que le va a gustar al usuario". } \\
\text { E3: "Para generar un buen producto tenés que jugar con la necesidad del usuario. No crear } \\
\text { cosas a la loca, porque vos podés crear y crear, pero si él no lo necesita, no lo va a usar". }\end{array}$ \\
\hline \multirow[b]{3}{*}{$\begin{array}{l}\text { Interacción de los } \\
\text { sujetos en ambientes } \\
\text { informáticos. }\end{array}$} & $\begin{array}{l}\text { Fuentes consulta- } \\
\text { das para conseguir } \\
\text { la información. }\end{array}$ & $\begin{array}{l}\text { Se revisan los sitios web oficia- } \\
\text { les de iOS y Android. Allí se } \\
\text { generan foros y tutoriales. }\end{array}$ & \\
\hline & $\begin{array}{l}\text { Acceso y } \\
\text { recopilación de la } \\
\text { información. }\end{array}$ & $\begin{array}{l}\text { Además de los sitios web ofi- } \\
\text { ciales, se revisan foros y wikis; } \\
\text { no solo los sitios registrados. } \\
\text { Los integrantes del semillero } \\
\text { buscan tutoriales en internet } \\
\text { (Youtube). } \\
\text { Se hacen revisiones de esta } \\
\text { información con el docente } \\
\text { líder del semillero. }\end{array}$ & $\begin{array}{l}\text { Son importantes los sitios web que no son sitios oficiales para comprender algunas formas } \\
\text { de realizar procedimientos. } \\
\text { E2: "En blogs o vídeos de Youtube uno se mete y pregunta: ‘¿cómo se hace tal cosa?' Y } \\
\text { ahí aparece, por ejemplo, un tutorial de eso. Entonces, es una experiencia en donde se va } \\
\text { construyendo como una gran cantidad de información en cuanto al tema”. } \\
\text { Los usuarios aprovechan las "librerías", que son fracciones de código que se pueden } \\
\text { reutilizar. } \\
\text { E2: "Lo que se está generando es una cadena en la que se a encuentra nueva información, } \\
\text { cosas que reinventan, cosas que se 'reúsan' y a partir de eso, es algo que va creciendo". }\end{array}$ \\
\hline & $\begin{array}{l}\text { Aspectos impor- } \\
\text { tantes de la inte- } \\
\text { racción a través } \\
\text { de los ambientes } \\
\text { informáticos. }\end{array}$ & $\begin{array}{l}\text { Los creadores de la app } \\
\text { destacan la rapidez que tiene } \\
\text { internet para el acceso a la } \\
\text { información. También acuden } \\
\text { a consultas en línea y a foros } \\
\text { para resolver dudas o preguntas } \\
\text { antes y durante las reuniones } \\
\text { grupales. }\end{array}$ & $\begin{array}{l}\text { Los integrantes del semillero destacan la forma como se puede encontrar gran cantidad de } \\
\text { información y modificar de manera constante. } \\
\text { E1: "Uno va y mira en internet porque alguien más ha preguntado lo que uno preguntó"... } \\
\text { El aprendizaje en materias teóricas... me parece genial porque es la rapidez o la facilidad } \\
\text { de acceder a cosas ahí, sin tener que ir a otro lugar" } \\
\text { E2: "Yo me acuesto a dormir y me pregunto: 'bueno, ’con qué me saldrán mañana?. La } \\
\text { virtualidad es algo que siempre está evolucionando". } \\
\text { Se genera una experiencia que va más allá del ámbito educativo. } \\
\text { E2: "Es una experiencia que no solo lo enriquece en el ámbito del conocimiento, sino } \\
\text { también en lo social, porque uno tiene la oportunidad de interactuar con otra gente que } \\
\text { está trabajando en aplicativos parecidos, en blogs o en videos de Youtube". } \\
\text { Es necesario filtrar la información porque no se puede confiar en todo lo que aparece en } \\
\text { la web. } \\
\text { E3: "A veces la información está muy dispersa o no está muy clara... Entonces, te toca } \\
\text { buscar mucho y no confiar en todo, porque hay gente que publica mucha basura. Lo más } \\
\text { complicado es encontrar información concreta". }\end{array}$ \\
\hline
\end{tabular}

Fuente: Elaboración propia. 
wikis. Estos lugares son creados de manera intersubjetiva mediante objetos virtuales como videos, textos o imágenes, y en ellos se encuentra gran variedad de información y la posibilidad de interactuar con otros que tienen los mismos gustos o inconvenientes. Aunque en la mayoría de los casos se da una comunicación sincrónica, no siempre es así debido a la gran cantidad de la interacción se produce a través de los elementos u objetos que se dejan de manera virtual en estos lugares.

Los miembros del semillero de investigación destacan la importancia de los grupos de desarrollo de software con los cuales interactúan a través de sitios web para intercambiar información o compartir librerías con el fin de resolver dudas o reportar nuevas funcionalidades de las aplicaciones. Estos elementos encontrados les permite seleccionar información y tomar elementos que posteriormente son llevados a las reuniones presenciales del semillero para ser discutidos entre los miembros del grupo y con su líder, quien ajusta los conceptos, resuelve las dudas que se generan y hace observaciones con el fin de solucionar los inconvenientes.

Asimismo, son conscientes de que son potentes alimentadores de datos y que la cantidad de información que fluye en las redes digitales no siempre es en un elemento que ayude a conseguir un buen resultado en las investigaciones. Es necesario filtrarla para transformarla en conocimiento y adaptarla al entorno, articulando de esta manera teoría y práctica.

\section{Análisis}

Se evidenció que en la construcción de objetos virtuales elaborados para ambientes informáticos, es importante tener en cuenta la interacción de los sujetos para seleccionar los elementos simbólicos, como la paleta de colores, la tipografía o la iconografía, con el fin de establecer una adecuada navegación, estructurar la aplicación y equilibrar la relación entre estos elementos para su posterior ubicación en la pantalla del ordenador, pues es a partir de los símbolos creados que se crean nuevos y se adaptan a los ya existentes.

Los constructores de objetos virtuales generan el diseño y establecen los elementos de acuerdo con las normas y características establecidas por cada sistema operativo, ya sea Android o iOS. Muchas de las especificaciones técnicas se encuentran documentadas en los sitios web oficiales, donde se incluyen foros para resolver dudas y un espacio exclusivo para developers. Se tiene, entonces, que este no es un proceso totalmente libre porque incluso los tamaños de los íconos y la tipografía dependen de los dispositivos móviles en los cuales se va a ejecutar la aplicación.

La interacción de los sujetos en los espacios digitales en los que obtienen los insumos para el análisis de los elementos gráficos que posteriormente incorporan en la aplicación, está influenciada por sitios web, blogs o wikis. En ellos acceden a videos, tutoriales o imágenes para encontrar información e interactuar con sujetos que tienen los mismos intereses. Sin embargo, en muchas ocasiones esta comunicación no se hace en tiempo real, razón por la cual los objetos virtualizados que circulan en estos sitios son importantes para hacer visible el conocimiento y transferir la información necesaria.

Se evidencia la importancia de las acciones que "los otros" ejecutan en el mundo digital para efectos técnicos y de efectividad de la aplicación, lo cual genera una preocupación constante por parte del grupo que elabora la interfaz gráfica. Este acercamiento a los demás, tiene el interés de lograr que el producto sea entendido por los usuarios, de ahí la importancia de ponerse en el lugar del otro (Miller, 1981). De esta manera, los sitios web oficiales de los diferentes sistemas operativos involucrados enfocan su discurso en lograr que los desarrollos se hagan técnicamente, por lo que se brinda prioridad a la técnica por encima de la creatividad o del concepto gráfico en la elaboración. Es, entonces, en el dibujo iconográfico que se evidencia la mayor libertad para desplegar la creatividad de los sujetos que las elaboran.

Los objetos virtuales no son objetos de aprendizaje como los proyecta la escuela, sino objetos informáticos que brindan la oportunidad de acceder a mucha información, lo que posibilita su diseminación. Estos sitios resultan relevantes para la creación de colectivos o grupos de redes aprendientes, lo que evidencia la interrelación entre grupos de sujetos y redes de información que, en últimas, convierte en un factor potencial de creación colectiva. Estos lugares híbridos y la mezcla de objetos y sujetos pugnan por cambios tecnológicos y la invención de nuevos objetos y son los usuarios de esta tecnología quienes a través de su interacción definen estos cambios, de la misma manera como ocurre en la sociedad.

Resulta importante la experiencia de la creación colectiva a través del semillero sobre la base de la interacción en ambientes informáticos, toda vez que brinda los elementos para ponderar aquello que la virtualidad le sugiere a la escuela como una oportunidad para potenciar grupos y construir conocimiento. 
La virtualización supone una relación entre los ámbitos público y privado en la manera como se construyen redes para reutilizar no solo información, sino también parte de objetos (librerías). Esta característica forma parte de los procesos de virtualización (efecto Moebius) y ofrece oportunidades para solucionar inconvenientes antes de que se presenten durante la puesta en marcha del aplicativo. Así, el trabajo en red proporciona otras maneras de acercarse al saber, acceder al aprendizaje, y gracias a la experiencia de la interacción abrir puertas al contacto con el mundo y diseñar formas de comprender la educación en los medios digitales.

\section{Discusión}

Basta reconocer los aspectos mediante los cuales un proceso se virtualiza para identificar que la virtualidad no depende exclusivamente de los dispositivos electrónicos. Un elemento puede ser virtual en un medio análogo y muchos de los procesos en dispositivos digitales pueden no ser virtuales (como potencia), sino una repetición o una selección de un elemento dentro de una lista de posibilidades. La subjetividad encuentra en la virtualidad nuevos elementos surgidos a raíz del ingreso de la tecnología web en la que el sujeto busca otras formas de agrupación social, lo que remite a pensar en la evolución de los entornos y los modos de vida que se suscitan en el mundo, de acuerdo con la conexión mediante dispositivos móviles, que logran que lo presencial o "en línea” tenga una nueva dimensión en los fenómenos comunicativos.

Estas formas de interacción que provee la tecnología a través de la informática y el desarrollo web, logran que los relatos hipertextuales fomenten la construcción de espacios públicos en internet e inviten a pensar otras herramientas para la creación o la inteligencia colectivas (Lévy, 2011). De esta manera, la virtualización genera tensiones en la escuela debido a que los procesos educativos a partir de clases virtuales proponen innumerables desafíos ante la cantidad de información almacenada en la red. Estas formas de enseñanza se mezclan con las prácticas educativas tradicionales y generan un instrumento que abre puertas a los procesos de construcción de conocimiento. Este cambio necesariamente implica una transformación en los procesos de construcción de conocimiento tanto en los sujetos, como en las instituciones, dado que las formas convencionales de originar y compartir los saberes en la actualidad generan desequilibrios en la escuela tradicional (Terrén, 1999).
Considerar estas opciones plantea reflexiones importantes acerca de la construcción de conocimiento y la forma de acceder a la información basado en la desescolarización, lo cual afecta el proceso educativo. Estas apreciaciones proponen una manera distinta de analizar las dinámicas que se generan en la escuela, pues si bien la educación es producto de la sociedad, los procesos que suceden a nivel de los asuntos educativos afectan lo social y a los sujetos (Martínez \& Orozco ,2010). Tal vez sea necesario preguntarse qué espera la educación de la virtualidad o qué distancia hay entre el sentido de la virtualidad y el de la educación, pues al parecer, resulta un reto virtualizar la comunicación en torno a la educación en el aula. Entonces, se convierte en una prioridad hacer que estos aspectos confluyan, pues la sociedad le pone retos a la escuela a través de la tecnología (MartínBarbero, 2009). Por lo tanto, conviene revisar la manera como estos ambientes, guiados por la escuela, resuelven los desafíos que la virtualidad plantea a la educación en relación con la forma como se comunican los sujetos por medio de los dispositivos electrónicos. La apuesta es lograr que los ambientes virtuales de aprendizaje no sean solo lugares para el intercambio de información, sino espacios que permitan a los sujetos aprender en el contexto de la vida cotidiana.

Reconocer la interacción entre los sujetos dentro de los ambientes informáticos mediante la tecnología web es importante para saber de qué manera definen sus acciones, pero también la forma como son influenciados por la experiencia que genera la interacción social y crea una influencia mutua (Ritzer, 1993). Por tal razón, es importante reconocer el significado y la interacción como procesos humanos en cuanto actos colectivos, sin olvidar que es el individuo quien realiza la interacción y el símbolo como elemento objetivado; es decir, debe tener un mismo significado para hacerse general (Carabaña \& Lamo de Espinoza, 1978).

Estos objetos facilitan la intersubjetividad en la virtualidad comoquiera que posibilitan la comunicación y aportan datos sobre los discursos sobre la base del aprendizaje fundamentado en la experiencia y llevado a cabo a lo largo y ancho de la vida. Un asunto que la Comisión Internacional para el Desarrollo de la Educación trabaja desde 1972 por encargo de la Unesco, bajo el concepto de "educación a lo largo de la vida" o "educación permanente" y en el que la educación no formal se incluye dentro de los procesos de aprehensión de conocimiento en la vida cotidiana, más allá de la idea de escolarización. Se enfatiza la importancia del sujeto y sus posibilidades de aprender 
según el entorno en el que se desarrolla y sus aptitudes personales (Morales, 2009).

De acuerdo con lo anterior, resulta complejo incluir estos procesos de interacción aún distantes de la educación formal, debido a una reificación de lo tecnológico que se enfoca en las herramientas digitales y deja de lado los procesos intersubjetivos y la reactualización de la experiencia en la virtualidad. Por tanto, este estudio muestra la conveniencia de indagar acerca de los elementos gráficos utilizados en los procesos de construcción de objetos virtuales, pues es a partir de ellos que se genera la interacción y se facilita la comunicación al destrabar los procesos inherentes a la experiencia social, pues son los usuarios quienes proveen sentidos a los íconos y hacen que la red continúe vigente. Esta validación social de lo gráfico institucionaliza nuevos relatos y potencia la virtualidad para mantener vigentes tanto los discursos como las instituciones.

Esta investigación deja abiertos básicamente dos interrogantes para futuras exploraciones. El primero tiene que ver con la manera como estos desarrollos técnicos se relacionan con los procesos estéticos en la creación de elementos icónicos dentro de la red, en virtud a la enorme cantidad de sitios gratuitos que proveen iconos o plantillas (themes) y otros elementos para la creación de interfaces gráficas de usuario. El segundo elemento se asocia con la forma de aprendizaje por medio de los objetos virtualizados (videos, imágenes, tutoriales) que se encuentran en internet (muchos forjados por los llamados youtubers) que buscan insertar información didáctica sobre elaboraciones o procedimientos técnicos. En este caso, es necesario hacer una revisión y comprender la aceptación y la efectividad de este tipo de narraciones que si bien se proyectan por fuera de la educación formal, proveen cambios importantes en la escuela tradicional aprovechando la tecnología web y los dispositivos móviles.

Agradecimientos: Al profesor Julián Humberto Arias Ph.D. por su apoyo constante.

\section{Referencias}

Alberdi, M. C. (2004). La imagen digital ¿̨nuevos interrogantes para la semiótica?. Artículo publicado en: La trama de la comunicación. Anuario del Departamento de Ciencias de la Computación, 9, 1-7.

Baudrillard, J. (1984). Las estrategias fatales. Barcelona, España: Editorial Anagrama.
Bautista, S., \& Méndez de Cuellar, M. (2015). Prácticas de lectura y escritura mediadas por las TIC en contextos educativos rurales. Revista Guillermo de Ockham, 13(1), 97-107.

Belvedere, C. (2013). El problema de la 'realidad' en el marco de la influencia prehispánica en la obra de Alfred Schutz. Investigaciones Fenomenológicas, 5, 245-277.

Berger, P., \& Luckmann, T. (2001). La construcción social de la realidad. Buenos Aires: Amorrortu Editores.

Blumer, H. (1982). El interaccionismo simbólico: perspectiva y método. Barcelona: Hora S.A.

Bonilla-Castro, E. \& Rodríguez, P. (1997). Más allá del dilema de los métodos. La investigación en Ciencias Sociales. Colombia: Uniandes.

Buján, F. (2010). Hacia una semiótica de las interfaces digitales: subjetividad e intersubjetividad en un entorno virtual de formación. Revista Estudios semióticos, 6(2), 40-48.

Carabaña, J., \& Lamo de Espinoza, E. M. (1978). La teoría social del interaccionismo simbólico: análisis y valoración crítica. Revista española de investigaciones sociológicas, 1, 159-203.

De la peña, F., \& Burgos, M. (2015). Modelo práctico de aplicación (app) para dispositivo móvil en las asignaturas universitarias de enseñanza a distancia. Experiencia con Android para la asignatura dirección de operaciones. Revista Electrónica de Tecnología Educativa, 51.

Giraldo, M. E. (2009). El interaccionismo simbólico, un enfoque metodológico para la investigación de las TIC en educación. Maestría en Educación, 1, 108-112.

González, M. \& Hernández, M. J. (2008). Interpretación de la virtualidad. El conocimiento mediado por espacios de interacción social. Revista Apertura, 8(9), 8-20.

Hernández, R.; Fernández, C. \& Baptista, M. (2010). Metodología de la investigación. Quinta edición. Mc Graw Hill.

Chun-Heng, H., \& Hou, C. (2015). Exploring the attractive factors of app icons. KSII Transactions on Internet and Information Systems, 9(6), 2251-2270. doi: 10.3837/ tiis. 2015.06 .016

Lévy, P. (2011). La esfera pública del siglo XXI. Techyredes, 8, 43-49.

Lévy, P. (1998). ¿Qué es lo virtual? Barcelona: Paidós.

Martín-Barbero, J. (1987). De los medios a las mediaciones. Comunicación, cultura y hegemonía. Barcelona: Gustavo Gili.

Martín-Barbero, J. (2009). Cuando la tecnología deja de ser una ayuda didáctica para convertirse en mediación cultural. Revista Electrónica Teoría de la Educación, 10(1), 19-31.

Martínez, A., \& Orozco, J. H. (2010). Políticas de escolarización en tiempos de multitud. Revista Educación y Pedagogía, 22 (88), 103-119. 
Morales, M. (Comp.). (2009). Aportes para la elaboración de propuestas de politicas educativas. Educación no formal. Una oportunidad para aprender. Uruguay: Unesco.

Roos, J., \& Gao, J. (2015). Overcoming the language barrier in mobile user interface design - A case study on a mobile health $a p p$. Australasian Conference on Information Systems, University of South Australia.

Tedesco, A. B., \& García, L. A. (2007). Tecnologías digitales en educación: ¿̨complacer o cruzar fronteras? Algunas ideas para el debate desde el paradigma crítico-reflexivo. Tecnología y Comunicación Educativas, 21(15), 86-100.

Terrén, E. (1999). Postmodernidad, legitimidad y educación. Revista Educação \& Sociedade, 20 (67), 11-47.

Virilio, P. (1997). El cibermundo, la politica de lo peor. Madrid: Cátedra. 